\title{
Meningioma como causa poco común de hiposmia. A propósito de un caso
}

\author{
Meningioma as an infrequent cause of hyposmia. Case report
}

Lorena Galindo-Iñiguez ${ }^{1,2}$, Karla Sanz-López ${ }^{3}$, Jesús Martínez-Alonso ${ }^{1,2}$.

\section{Resumen}

La afectación del sentido del olfato puede pasar desapercibida en gran parte de los pacientes, siendo únicamente valorada cuando la alteración es importante. Sin embargo, la hiposmia es un síntoma asociado a numerosas patologías, de menor a mayor gravedad. Este sentido está relacionado con nuestros recuerdos y puede alertarnos de potenciales amenazas. Como médicos especialistas en Otorrinolaringología debemos estar alerta ante determinados síntomas que orienten a posible etiología central. Presentamos el caso de una mujer de 39 años que acudió al Servicio de Otorrinolaringología del Hospital General Universitario Morales Meseguer derivada por hiposmia de un año de evolución con cervicalgia y cefalea occipital progresiva, sin otra sintomatología asociada. La exploración otorrinolaringológica resultó normal. Debido a la persistencia sintomática se solicitó una tomografía computarizada de senos paranasales con hallazgos de masa intracraneal extraaxial compatible con meningioma de fosa craneal anterior.

Palabras clave: hiposmia, otorrinolaringología, meningioma.

\begin{abstract}
The affectation of smell can go unnoticed in a great part of the patients, being only valued when the alteration is important. However, this sense is related to our memories and can alert us to potential threats. As otolaryngologists we must be alert to certain symptoms that may lead to a possible central aetiology. We present the case of a 39-year-old woman who went to the otolaryngology service at Morales Meseguer General University Hospital due to one-year evolution of hyposmia with cervicalgia and progressive occipital headache, with no other symptoms associated. The otolaryngology examination was anodyne. In view of symptomatic persistence, a paranasal sinus computed tomography scan was requested with findings of extra-axial intracranial mass compatible with anterior cranial fossa meningioma.
\end{abstract}

Keywords: hyposmia, otolaryngology, meningioma.

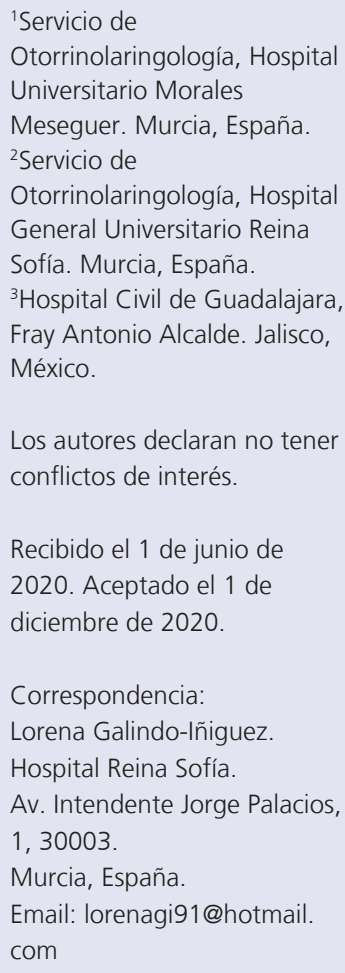

Correspondencia: Lorena Galindo-Iñiguez. Hospital Reina Sofía. Av. Intendente Jorge Palacios, 1, 30003

Murcia, España.

Email: lorenagi91@hotmail. com

\section{Introducción}

La alteración del olfato es un síntoma asociado a numerosas patologías, de diferente gravedad. La mayoría de los pacientes no suelen consultar por hiposmia a menos que esté asociado a otra sintomatología como obstrucción nasal y cefalea, entre otras ${ }^{1}$. Así, hasta el $74,2 \%$ de los pacientes no reconocen el déficit. Por tanto, se sugiere que la mayoría pueden no ser diagnosticados ni tratados ${ }^{2}$. La sensibilidad de los informes puede mejorarse realizando preguntas más detalladas como las que se incluyen en la encuesta del National Health and Nutritional Examination Survey (NHANES), (Tabla 1) $)^{3}$. Una respuesta positiva a cualquiera de estas preguntas puede considerarse una posible deficiencia olfativa.

\section{Etiología}

La causa más frecuente de pérdida de olfato es la congestión de la mucosa nasal consecuencia de infecciones virales, generalmente un resfriado común casi siempre reversible, pero 
Tabla 1. Encuesta NHANES ${ }^{3}$

Durante los últimos 12 meses, ¿ha tenido algún problema con su capacidad para oler, como no poder oler las cosas o que no huelan como se supone que deben?

¿Calificaría su capacidad para oler ahora en comparación a antes? ¿Es mejor ahora, peor ahora, o no hay cambios?

¿Huele un olor desagradable, malo o a quemado?

en general, cualquier proceso que produzca obstrucción de las fosas nasales puede cursar con hiposmia o anosmia. Tras la etiología inflamatoria, la segunda causa más frecuente de hiposmia es la etiología posinfecciosa. Ac-

Tabla 2. Etiología de trastornos del olfato ${ }^{6}$

Drogas y medicamentos

Endocrinas: síndrome de Cushing, hipotiroidismo, pseudoparatiroidismo, insuficiencia adrenocortical

Sustancias industriales (polvos, metales): acetona, benceno, cromo, disolventes

Enfermedades nasosinusales: rinosinusitis, poliposis nasal, posinfecciosa, postraumática

Tumorales: intracraneales (meningioma, glioma), intranasales (estesioneuroblastoma, adenocarcinoma)

Enfermedades neurológicas: Alzheimer, Parkinson

Psiquiátricas

Nutricionales y metabólicas

\section{Tabla 3. Agentes químicos que alteran el sentido del} olfato ${ }^{7}$

\begin{tabular}{ll}
\hline Agente & Mecanismo \\
\hline Tabaco & Daño a la mucosa nasal \\
\hline Cadmio & Toxicidad celular \\
\hline Cocaína & Microinfarto \\
\hline Diltiazem & Afecta a la transmisión sensitiva \\
\hline Metotrexato & $\begin{array}{l}\text { perjudica el crecimiento y la sustitución } \\
\text { de las células de la mucosa }\end{array}$ \\
\hline Nifedipino & Afecta a la transmisión sensitiva \\
\hline Nitrato de plata & Depósitos de plata en el tejido \\
\hline
\end{tabular}

tualmente la anosmia se ha notificado como un síntoma de presentación en algunos pacientes a los que finalmente se les diagnosticó COVID-194. Otras condiciones que pueden conducir a hiposmia son alergias, antecedentes de cirugía nasal y/o traumatismos craneoencefálicos e historia de poliposis nasosinusal, pero también deben tenerse en consideración otras etiologías como enfermedades psiquiátricas y lesiones del sistema nervioso central ${ }^{5}$ (Tabla 2$)^{6}$.

\section{Diagnóstico}

La evaluación de los pacientes con anomalías en el olfato requiere de una anamnesis detallada. Resulta de especial relevancia documentar el momento de inicio de los síntomas. Así mismo, los síntomas asociados como la cefalea y los trastornos del comportamiento deben hacernos sospechar patología del sistema nervioso central ${ }^{5}$. Los antecedentes personales como el uso de tabaco y alcohol, las posibles exposiciones ocupacionales y el uso de fármacos deben ser evaluados (Tabla 3$)^{7}$.

Para evaluar la hiposmia se pueden utilizar diferentes pruebas que determinan el umbral olfatorio y pruebas de discriminación olfativa. Existen varios test olfatorios actualmente en uso, de los cuales destaca el UPSIT (Test de investigación del olfato de la Universidad de Pennsylvania) que evalúa cuarenta fragancias, y el Sniffin Stick Test, que consiste en la presentación de doce odorantes y cuyo valor será considerado anormal cuando el sujeto reconozca menos de diez olores. Sin embargo, esto varía según el sexo y la edad del paciente; siendo mejor en mujeres y población joven? ${ }^{7}$. Determinados estudios de laboratorio como 
la detección de anticuerpos contra Ro/SSA y LA/SSB puede ser valiosa para identificar a los pacientes con síndrome de Sjögren, mientras que la medición de concentraciones de metales pesados puede estar justificada en pacientes seleccionados con hiposmia asociada a disgeusia. El algoritmo de estudio incluye una anamnesis detallada y exploración con rinoscopia anterior y videonasofaringoscopia. Se pedirán estudios de imagen en pacientes con alteraciones neurológicas a la exploración, antecedentes de traumatismo craneoencefálico o sospecha de lesión asociada nasosinusal o del sistema nervioso central ${ }^{8}$. La tomografía computarizada resulta una buena opción para evaluar la afectación ósea, la base del cráneo y la patología nasosinusal. La resonancia magnética constituye una alternativa para evaluar tejidos blandos, incluyendo los senos paranasales. Hoekman y cols. ${ }^{8}$ presentaron un estudio retrospectivo de cohorte de 839 pacientes con hiposmia, realizando una resonancia magnética en el 55\% de la muestra. Tan sólo el 0,8\% de las resonancias magnéticas documentaron anomalías que explicaran su pérdida olfativa, por lo que determinaron que en la mayoría de los pacientes con hiposmia no se deben realizar pruebas de imagen de forma rutinaria. La decisión de realizar pruebas de imagen depende de la anamnesis y exploración física. Existen diferentes algoritmos diagnósticos en el estudio de pacientes con hiposmia (Figura 1$)^{8}$.

\section{Caso Clínico}

Presentamos a una mujer de 39 años que acudió al Servicio de Otorrinolaringología del Hospital General Universitario Morales Meseguer, sin antecedentes personales de interés, no presentaba consumo de drogas vía nasal, ni tenía antecedentes familiares de enfermedades neurodegenerativas. La paciente presentaba hiposmia progresiva, cervicalgia y cefalea occipital de un año de evolución, sin asociar parosmias ni disosmias, ni referir disgeusia.

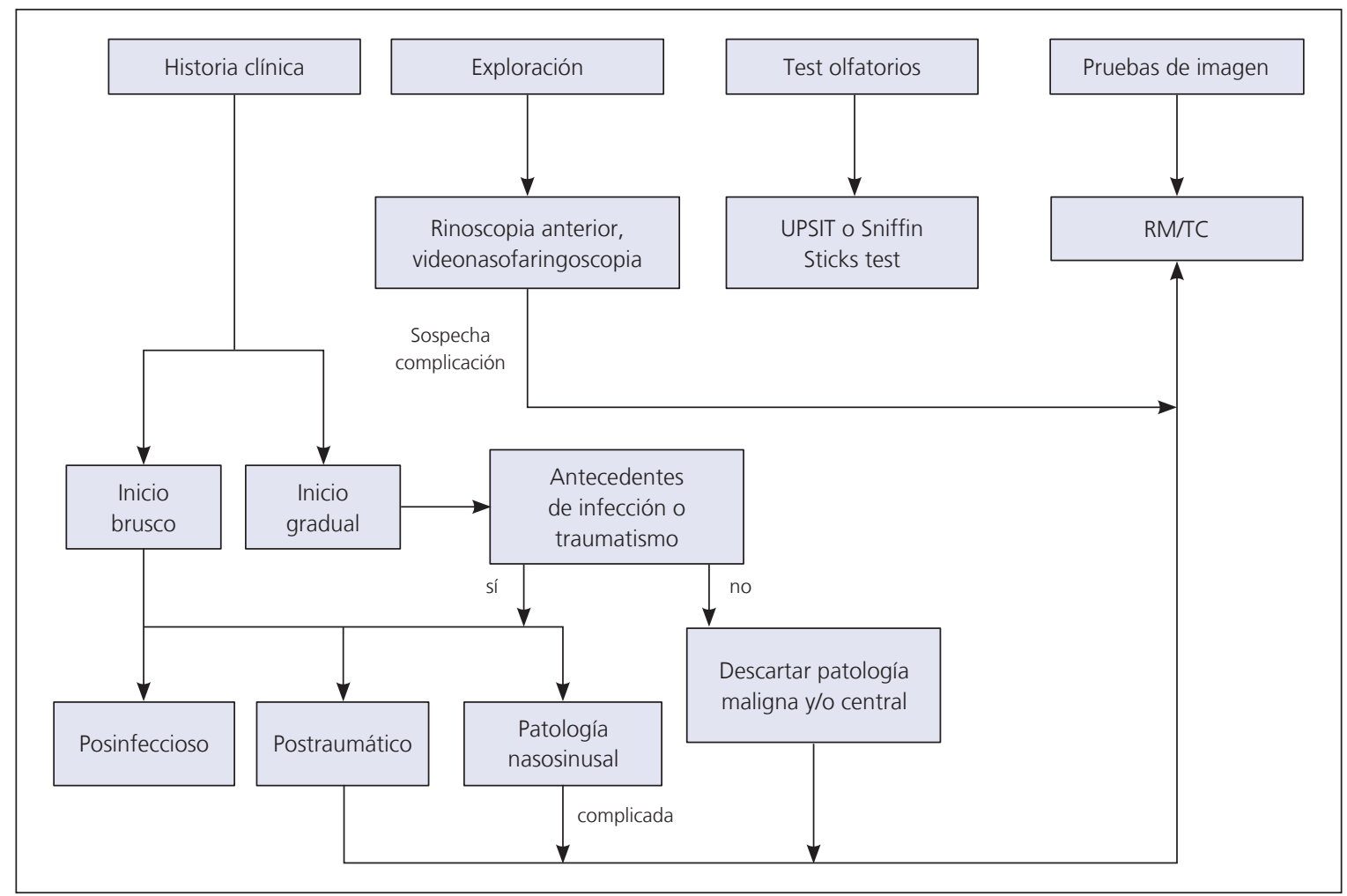

Figura 1. Algoritmo diagnóstico en pacientes con hiposmia 
La exploración resultó normal. Debido a la persistencia sintomática se realizó el Sniffin Stick Test y se solicitó una tomografía computarizada de senos paranasales con hallazgos de masa intracraneal extraaxial compatible con meningioma (Figura 2).

Ante los hallazgos se solicitó resonancia magnética (RM) (Figura 3) con hallazgos de meningioma frontobasal derecho isointenso, que se extiende a la lámina cribosa con her-

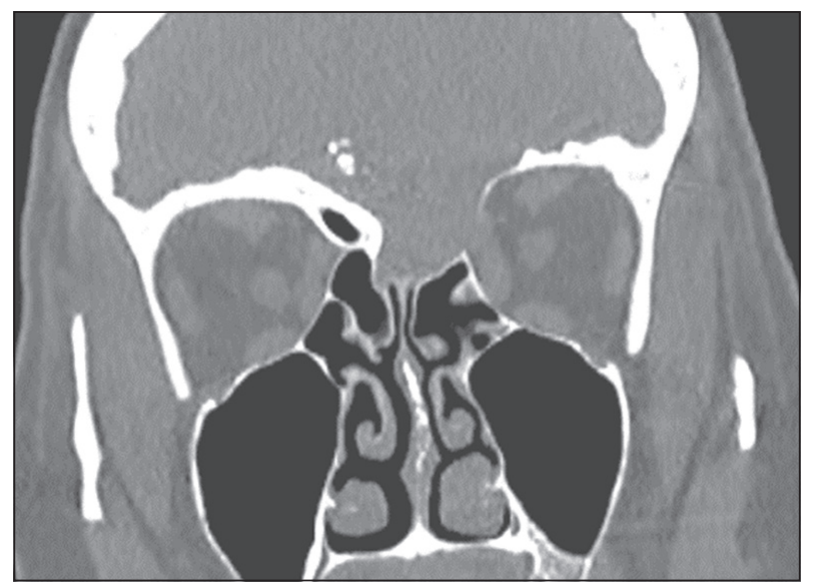

Figura 2. Tomografía computarizada de cavidades paranasales. Masa intracraneal extraaxial hiperdensa en fosa craneal anterior de $7,5 \mathrm{~cm}$ en relación con probable meningioma con lesión de lámina papirácea.

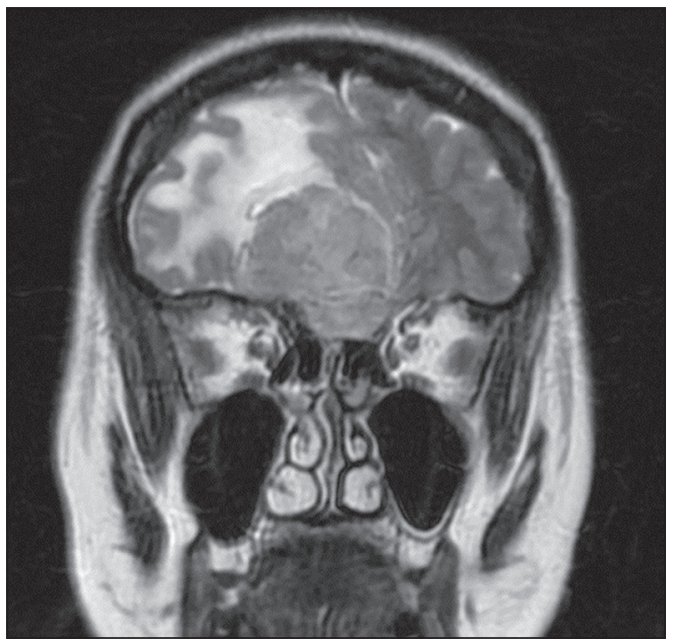

Figura 3. Resonancia magnética craneal. Meningioma de estirpe transicional frontobasal derecho que se extiende a la lámina cribosa con herniación del lóbulo frontal derecho hacia la silla turca e hidrocefalia obstructiva de ambos ventrículos laterales. niación hacia la silla turca, del lóbulo frontal derecho e hidrocefalia obstructiva de ambos ventrículos laterales, por lo que se derivó al Servicio de Neurocirugía de referencia.

La paciente se intervino mediante craneotomía bicoronal y exéresis completa de lesión expansiva compatible con meningioma del surco olfatorio, extirpándose el bulbo olfatorio afectado. Durante la cirugía se evidenció infiltración de la duramadre frontal bilateral, sustituyendo la misma por un parche. El resultado final anatomopatológico fue compatible con meningioma meningotelial (grado I de la clasificación de la Organización Mundial de la Salud, OMS). Tras un año de evolución la paciente se encuentra con buen estado general, sin cefalea ni otra sintomatología neurológica, excepto anosmia (Figura 4).

\section{Discusión}

El caso presentado permitió afianzar nuestro aprendizaje en el manejo de pacientes con alteraciones del olfato. A pesar de considerar la encuesta NHANES ${ }^{3}$, no se realizó una anamnesis acerca de la presencia de disosmia o parosmia así como tampoco se realizó test diagnóstico de olfato hasta que la paciente consultó en una segunda ocasión, pudiendo

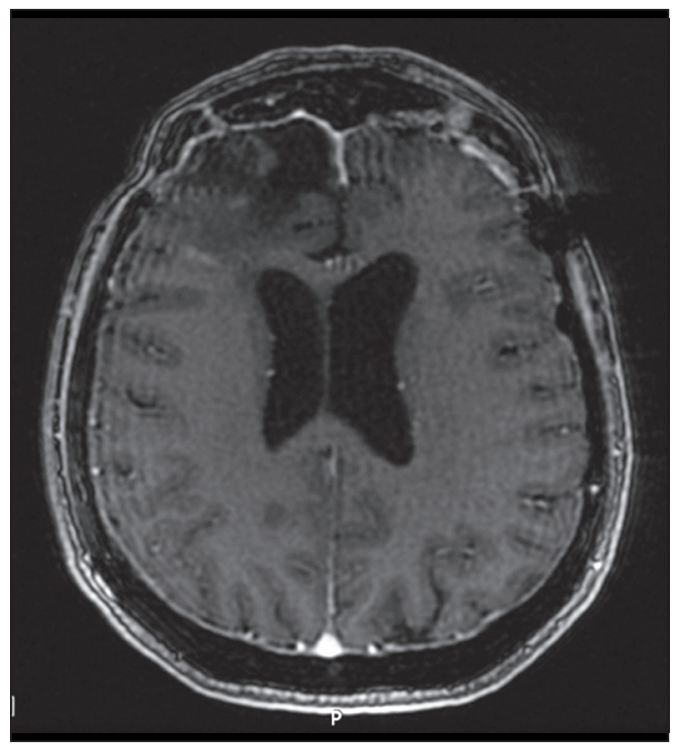

Figura 4. Resonancia magnética craneal posquirúrgica. 
haberse realizado con anterioridad. Consideramos que el especialista en otorrinolaringología debe prestar especial atención y realizar un estudio detallado según un algoritmo diagnóstico ante determinados síntomas que puedan orientar a patología de mayor gravedad, tales como lesiones del sistema nervioso central. Los meningiomas son tumores generalmente benignos, de lento crecimiento, que se originan en las células meningoepiteliales de la membrana aracnoidea. Suponen entre el $15 \%$ y el $20 \%$ de los tumores intracraneales aproximadamente, siendo los tumores intracraneales primarios más frecuentes, con una mayor incidencia en la edad media y en el sexo femenino ${ }^{8}$.

Factores genéticos y ambientales se han implicado en su formación; sin embargo, la mayoría de los casos tiene un origen idiopático ${ }^{9}$. Se ha postulado el papel etiológico de las radiaciones ionizantes, los traumatismos y las hormonas, pudiendo además aparecer en el seno de síndromes familiares hereditarios. Entre las alteraciones genéticas, la más frecuente es la monosomía del cromosoma 22 y la deleción del cromosoma 22 en asociación con la neurofibromatosis tipo $2^{9}$. Dentro de los meningiomas intracraneales, aquellos que afectan a la base del cráneo alcanzan aproximadamente el 25\%-35\% de los casos $^{8}$. Dentro de los meningiomas de la fosa anterior, se han considerado tres localizaciones: meningiomas de la órbita, surco olfatorio y del tubérculo selar ${ }^{10}$. La clínica de cada localización dependerá de la afectación peritumoral $^{11}$. El diagnóstico se realiza a través de exámenes de neuroimagen. En la tomografía computarizada aparecen isodensos $(40 \%)$ o discretamente hiperdensos (60\%), que se refuerzan homogéneamente con el contraste, generalmente con edema perilesional. En la resonancia magnética aparecen como isointensos o moderadamente hipointensos en T1. A veces son de aspecto heterogéneo, por la presencia de vasos intratumorales ${ }^{11}$. Desde el punto de vista anatomopatológico, existen diversas variedades entre las que se encuentran la variedad meningoepitelial, fibroblástica, psamomatosa, transicional o mixta, meningiomas con marcada vascularización, meningiomas papilares y anaplásicos ${ }^{11}$. Actualmente se utiliza el esquema de clasificación propuesto por la OMS edición $2007^{12}$ que reconoce 15 variantes agrupadas en tres grados: el grado I corresponde a las formas clásicas o benignas e incluye nueve subtipos, el grado II y el grado III incluyen cada una tres subtipos diferentes y representan las formas atípicas y las malignas ${ }^{13}$. Los meningiomas del surco olfatorio representan entre el $8 \%$ y el $13 \%$ de todos los meningiomas intracraneales. Entre las manifestaciones sintomáticas podemos encontrar crisis epilépticas, déficit sensitivo-motores, trastornos del comportamiento y trastornos del equilibrio, entre otras ${ }^{14}$. la clínica se relaciona con la localización del tumor y con su tamaño, siendo la manifestación sintomática en ocasiones inespecífica ${ }^{13}$. El tratamiento de estos tumores es quirúrgico, con lo que se consigue la mayoría de las veces la curación del paciente $^{14}$. Los abordajes endoscópicos reducen la manipulación y permiten una recuperación rápida del paciente, con mejores resultados estéticos. Sin embargo, entre las limitaciones del abordaje endoscópico se encuentran la necesidad de instrumental y otras limitaciones determinadas por la extensión y tamaño tumoral ${ }^{14}$. En el caso de nuestra paciente se optó por abordaje mediante craneotomía bicoronal y exéresis completa de la lesión debido al tamaño tumoral y a no considerar posible la extirpación tumoral en una sola pieza sin lesionar otras estructuras. Aunque la mayoría de meningiomas tienen un comportamiento benigno, tienen la capacidad de evolucionar y progresar a un grado histológico mayor (atípico y anaplásico) con comportamiento más agresivo y mayor tasa de recurrencias. Los meningiomas grado II (atípico, de células claras y cordoide) presentan un índice de recurrencia a cinco años del $40 \%$ después de la resección total en comparación con $5 \%$ de los meningiomas benignos tratados de la misma manera ${ }^{15}$. Así mismo, los meningiomas grado III (anaplásico, papilar y rabdoide) se asocian con un comportamiento clínico más agresivo, con mayor probabilidad de diseminación y peor pronóstico. Entre las opciones terapéuticas la radioterapia representa una alternativa de tratamiento para frenar el crecimiento de meningiomas pequeños localizados en regiones de difícil acceso (seno cavernoso) o que engloban estructuras vasculares importantes (tercio medio de ala esfenoidal). 


\section{Conclusión}

La alteración del olfato es un síntoma común cuyo diagnóstico diferencial incluye multitud de patologías de diferente gravedad. El caso presentado permitió afianzar nuestro aprendizaje en el manejo de pacientes con alteraciones del olfato, destacando la importancia de realizar un estudio detallado de los pacientes que consultan por este motivo, según el algoritmo diagnóstico presentado. Los meningiomas representan las neoplasias benignas más frecuentes del sistema nervioso central y en la mayoría de los casos se corresponden con tumores grado I en la clasificación de la OMS, por lo que se consideran generalmente benignos y el tratamiento quirúrgico consigue en la mayoría de las veces buenos resultados. Sin embargo, pueden causar morbimortalidad por efecto de masa y destrucción del parénquima adyacente, por lo que la actitud de sospecha en estos casos es fundamental para afrontar un manejo adecuado.

\section{Bibliografía}

1. Boesveldt S, Postma EM, et al. Anosmia-A Clinical Review. Chem Senses. 2017;42(7):513-523. doi: 10.1093/chemse/bjx025.

2. Adams DR, Wroblewski KE, et al. Factors Associated with Inaccurate Self-Reporting of Olfactory Dysfunction in Older US Adults. Chem Senses. 2017;42(3):223-231. doi: 10.1093/chemse/bjw108.

3. Hoffman HJ, Rawal S, et al. New chemosensory component in the U.S. National Health and Nutrition Examination Survey (NHANES): first-year results for measured olfactory dysfunction. Rev Endocr Metab Disord. 2016;17(2):221-40. doi: 10.1007/s11154-0169364-1.

4. Lechien JR, Chiesa-Estomba CM, et al. Olfactory and gustatory dysfunctions as a clinical presentation of mild-to-moderate forms of the coronavirus disease (COVID-19): a multicenter European study. Eur Arch Otorhinolaryngol. 2020;277(8):2251-2261. doi: 10.1007/s00405-020-05965-1.

5. Bayley P, Bucy PC. The origin and nature of meningeal tumors. Am J Cancer. 1931; 15:15-54.

6. Frye RE, Schwartz BS, et al. Dose-related effects of cigarette smoking on olfactory function. JAMA. 1990;263(9):1233-6.

7. Hummel T, Sekinger B, et al. 'Sniffin' sticks': olfactory performance assessed by the combined testing of odor identification, odor discrimination and olfactory threshold. Chem Senses. 1997;22(1):39-52. doi: 10.1093/chemse/22.1.39.

8. Hoekman PK, Houlton JJ, et al. The utility of magnetic resonance imaging in the diagnostic evaluation of idiopathic olfactory loss. Laryngoscope. 2014;124(2):365-8. doi: 10.1002/lary.24248.

9. Malaty J, Malaty IA. Smell and taste disorders in primary care. Am Fam Physician. 2013;88(12):852-9.

10. Solero CL, Giombini S, et al. Suprasellar and olfactory meningiomas. Report on a series of 153 personal cases. Acta Neurochir (Wien). 1983;67(3-4):181-94. doi: 10.1007/BF01401420.

11. Riemenschneider MJ, Perry A, et al. Histological classification and molecular genetics of meningiomas. Lancet Neurol. 2006;5(12):1045-54. doi: 10.1016/ S1474-4422(06)70625-1.

12. Louis DN, Perry A, et al. The 2016 World Health Organization Classification of Tumors of the Central Nervous System: a summary. Acta Neuropathol. 2016;131(6):803-20. doi: 10.1007/s00401-016-1545-1.

13. Burger PC, Scheithauer BW, et al. Surgical pathology of the nervous system and its coverings. 4 th ed. New York. Churchill Livingstone, 2002: 49-71.

14. Snyderman $\mathrm{CH}$, Pant $\mathrm{H}$, et al. What are the limits of endoscopic sinus surgery?: the expanded endonasal approach to the skull base. Keio J Med. 2009;58(3):152-60. doi: 10.2302/kjm.58.152.

15. Perry A, Brat DJ, et al. Meningiomas. In: Practical Surgical Neuropathology. Ed. Churchill Livingstone, 2010: pp 185-217. 